\title{
Shared-by-Wavelength-Switches: A Node Architecture Using Small Optical Switches and Shared Wavelength Converters
}

\author{
Tony K. C. Chan, Eric W. M. Wong, Senior Member, IEEE, and Yiu-Wing Leung, Senior Member, IEEE
}

\begin{abstract}
Wavelength converters can significantly improve the blocking performance of all-optical networks. The existing node architectures require large optical switches for sharing the wavelength converters. This letter proposes a new node architecture called shared-by-wavelength-switches. This node architecture requires significantly smaller and cheaper optical switches for sharing the wavelength converters while it gives nearly the same blocking performance as the existing node architectures.
\end{abstract}

Index Terms-All-optical networks, optical switches, wavelength converters.

\section{INTRODUCTION}

W AVELENGTH converters can significantly improve the blocking performance of all-optical networks [1]-[8]. It is necessary to design node architectures for supporting wavelength conversion. The simplest node architecture, called dedicated wavelength-convertible switch [1], places one wavelength converter on each outgoing channel prior to multiplexing. Using this architecture, the expensive wavelength converters cannot be shared [1]. Hence, several node architectures with shared wavelength converters were proposed [1]-[3] such as share-per-node (SPN) and share-per-link, and they still receive much attention today [5]-[8]. Fig. 1 shows the architecture of SPN. SPN was demonstrated to achieve nearly the same blocking performance as the dedicated wavelength-convertible switch [1].

The existing node architectures require large optical switches for sharing wavelength converters, especially when each fiber provides many wavelength channels via dense wavelength-division multiplexing (some commercial optical systems support up to 160 wavelength channels per fiber [9]). For example, if a node using the SPN architecture (see Fig. 1) has $F$ incoming/outgoing fibers and with $W$ wavelength channels per fiber, it requires an $F W \times(F W+M)$ optical switch, where $M$ is the number of wavelength converters per node. If $F=10, W=$ 160 , and $M=20$, the node requires a $1600 \times 1620$ optical switch. This results in two difficulties:

Manuscript received December 16, 2005; revised March 5, 2006. This work was supported by a grant from the Research Grants Council of the Hong Kong Special Administrative Region, China (Project 9040928) and by a grant from the HKBU FRG.

T. K. C. Chan and E. W. M. Wong are with the Department of Electronic Engineering, City University of Hong Kong, Kowloon Tong, Hong Kong (e-mail: tkcchan@cityu.edu.hk; ewong@ee.cityu.edu.hk).

Y.-W. Leung is with the Department of Computer Science, Hong Kong Baptist University, Kowloon Tong, Hong Kong (e-mail: ywleung @ comp.hkbu.edu.hk). Digital Object Identifier 10.1109/LPT.2006.877009

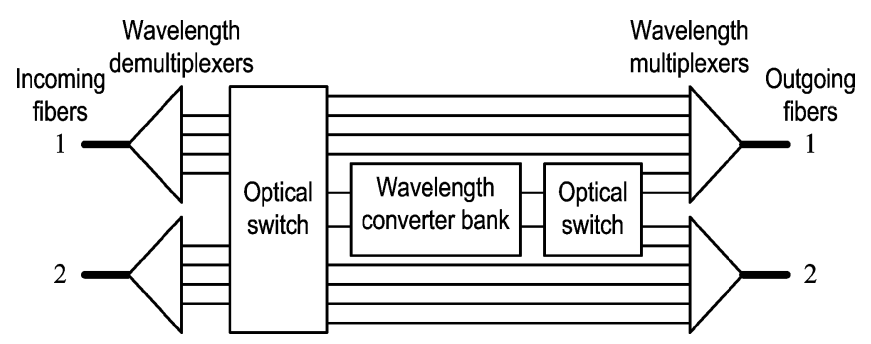

Fig. 1. SPN: an existing node architecture [1].

- It is difficult to realize large optical switches because of various technological constraints (e.g., insertion loss, crosstalk, optical fiber bundling, mirror manufacturing, reliability, optical packaging, and control complexity [10]-[12]). For example, the three-dimensional microelectromechanical system (3-D MEMS) is the most promising technology for realizing large optical switches [11]-[13] but the largest available switch size is only $1024 \times 1024$ [11].

- The cost of an optical switch increases exponentially with the switch size because 1) the number of switch elements required increases rapidly [14], 2) higher technological requirements are involved in realizing large optical switches (e.g., smaller loss per switch element, a higher precision requirement on the tilting motion of the mirrors for 3-D MEMS, etc.) [10], [12], and 3) a large optical switch involves more complex control and interconnections [10], [12]. Therefore, large optical switches are much more expensive than small ones.

To tackle the above difficulties, we propose a new node architecture called shared-by-wavelength-switches. This node architecture only requires small and cheap optical switches for sharing the wavelength converters but it can achieve nearly the same blocking performance as the SPN architecture. In addition, this node architecture can complement the novel two-layer wavelength conversion scheme [7].

\section{SHARED-BY-WAVELENGTH-SWITCHES}

Our main ideas are as follows. We adopt $W$ optical switches of smaller size for $W$ wavelength channels, respectively. When an incoming channel at any wavelength must go through wavelength conversion, this channel is switched to one of the shared wavelength converters. In this manner, the node requires smaller optical switches while the incoming channels can 


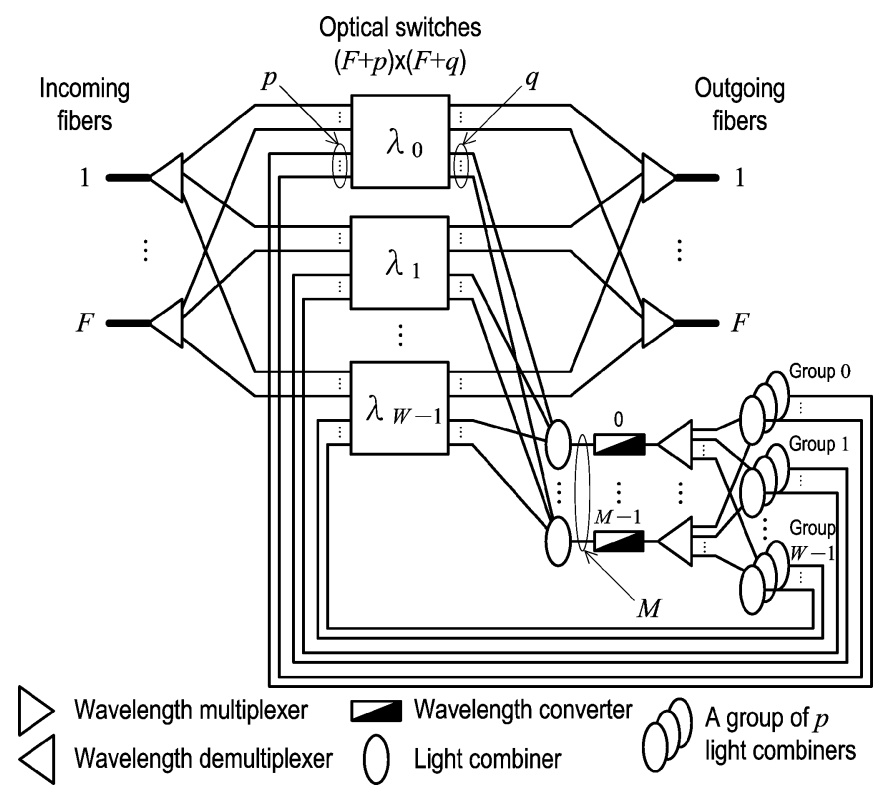

Fig. 2. Shared-by-wavelength-switches: the proposed node architecture.

share the expensive wavelength converters. At the same time, we design the interconnections within the node such that the resulting node achieves nearly the same blocking probability as the existing nodes. This new node architecture is named shared-by-wavelength-switches because each wavelength converter can be shared by the wavelength switches (i.e., optical switches at different wavelengths).

Fig. 2 shows our proposed design which requires $W$ pieces of $(F+p) \times(F+q)$ optical switches, $M$ wavelength converters, $F+M$ demultiplexers, $F$ multiplexers, and $M+p W$ light combiners. $p$ and $q$ are design choices, where $p$ represents the maximum number of light beams that can be converted to each wavelength and $q$ represents the maximum number of light beams from each optical switch that can undergo wavelength conversion. Each optical switch is responsible for switching the data on one wavelength, and each wavelength converter can convert the incoming light beam into any other wavelengths. The light combiners are passive devices which can combine multiple inputs into one output. The $M$ light combiners before the wavelength converters combine the outputs of different optical switches to the wavelength converters, while the other $p W$ light combiners combine the demuliplexed outputs back to the optical switches. All light combiners are allowed at most one light beam passing through each of them.

We connect the light combiners in a cyclic manner, such that each light combiner before the wavelength converters would have about $q W / M$ inputs and each of the other light combiners would have about $M / p$ inputs. To describe the connections of the light combiners, we need the following numbering convention (see Fig. 2):

- The $q$ outputs of each optical switch $w(0 \leq w \leq W-1)$ are numbered $w q, w q+1, \ldots, w q+q-1$.

- The $M$ light combiners connected before the wavelength converters are numbered $0,1, \ldots, M-1$, and the $M$ demultiplexers connected after the wavelength converters are numbered in a similar manner.
- The $p W$ light combiners connected after the demultiplexers are divided into $W$ group, and the $p$ light combiners of each combiner group are numbered $0,1, \ldots, p-1$.

For the first $M$ light combiners, we connect output $i$ of the optical switches to the light combiner $(i \bmod M)$ and the output of each light combiner is connected to one wavelength converter.

For the other $p W$ light combiners, each combiner group is responsible for one wavelength. For each combiner group $w$ $(0 \leq w \leq W-1)$, the output of demultiplexer $m$ on wavelength $\lambda_{w}$ is connected to the light combiner $(m \bmod p)$ and the outputs of the light combiners are connected to optical switch $w$.

\section{DISCUSSION}

Our proposed node architecture can achieve a very favorable tradeoff: its complexity can be significantly reduced while its performance only slightly degrades. The network designers can vary $p$ and $q$ to make this tradeoff to fulfill their preference requirement. In particular, we demonstrate in Section IV that this tradeoff is very favorable. On the other hand, the existing node architectures cannot provide this tradeoff.

When $p$ and $q$ equal $M$, the blocking conditions of our design are the same as those of SPN. When $p$ and $q$ are smaller than $M$, a request for a lightpath at wavelength $\lambda_{w}$ would be blocked under the following conditions: 1) wavelength $\lambda_{w}$ of the destined outgoing fiber of this request is engaged and 2) all the $q$ groups of paths associated with the $q$ wavelength converters connected from the optical switch at wavelength $\lambda_{w}$ are not available, where a group of paths associated with a particular wavelength converter are not available if either this wavelength converter is engaged or the output paths from this wavelength converter to the destined fiber are engaged.

Compared with other existing designs [1]-[3], our design requires much smaller and cheaper optical switches (and some inexpensive and passive light combiners [13]) to give nearly the same blocking probability.

\section{Simulation Results}

To compare our proposed design with SPN, we adopt the same routing algorithm as in [1]. This algorithm transforms the network into a directed graph with weighted edges, and employs Dijkstra's algorithm to determine the routes. Whenever a connection is set up or torn down, the weight of all related edges will be updated according to the connection and the node architecture. Hence, the routes of all call requests can be adaptively determined.

We adopt the ARPA2 network [1] with 21 nodes and 26 bidirectional links. The call requests of each node follow a Poisson process with arrival rate $r$, and the duration of each call request is exponentially distributed with rate $\mu$. Whenever a call request cannot be served, it is blocked. Each simulation experiment is run until the $95 \%$ confidence interval of the blocking probability is sufficiently small, where the blocking probability is the probability that an end-to-end lightpath for one source-destination pair is blocked. 


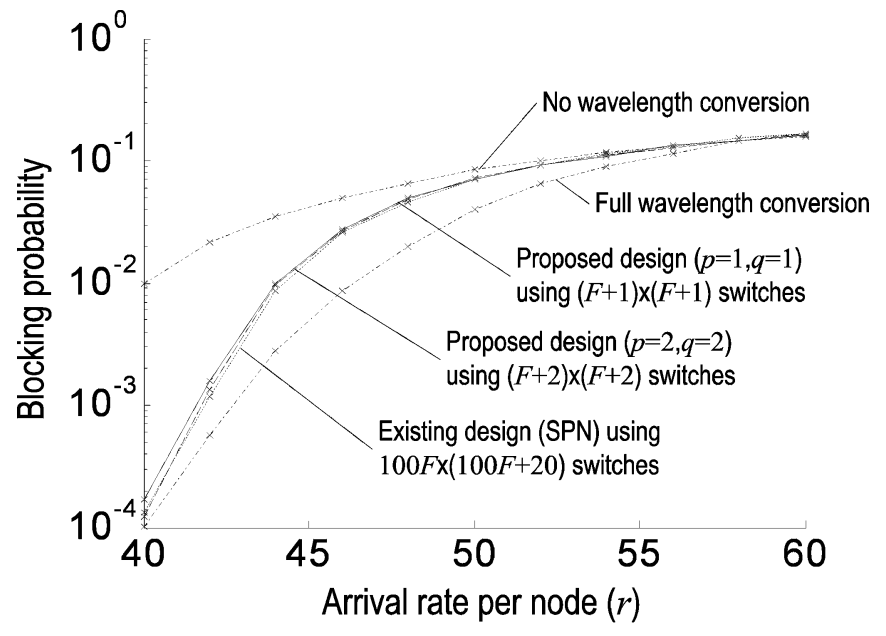

(a)

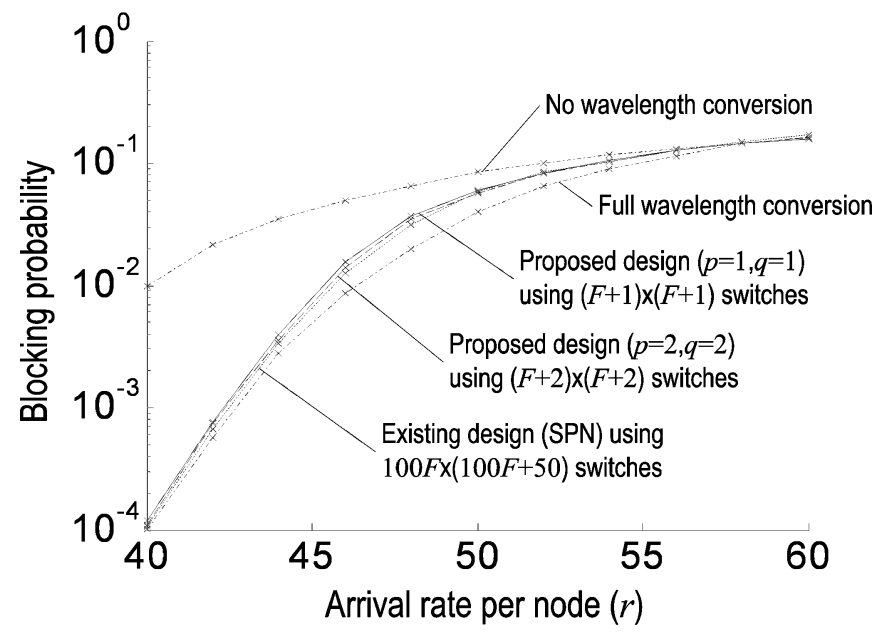

(b)

Fig. 3. Blocking probability versus traffic loading for different node architecture designs at $W=100$ and $\mu=1$. (a) Number of converters per node $M=20$. (b) Number of converters per node $M=50$.

Fig. 3 shows the blocking probability versus traffic load for $W=100$ and $\mu=1$. We see that the proposed node architecture requires much smaller and hence cheaper optical switches than the SPN architecture but it gives nearly the same blocking performance as SPN. For example, when $M=50$ and $r=40$, our node architecture (with $p=1$ and $q=1$ ) and the SPN architecture have blocking probabilities $1.18 \times 10^{-4}$ and $1.06 \times 10^{-4}$, respectively (i.e., nearly the same blocking performance), but our node architecture only requires $5 \times 5$ optical switches while the SPN architectures requires $400 \times 450$ optical switches for $F=4$.

Fig. 3 also demonstrates that only a few shared wavelength converters can significantly improve the blocking performance. For example, the blocking probability of no wavelength conversion at $r=40$ equals $9.98 \times 10^{-3}$. With 20 shared wavelength converters (i.e., $M=20$ ), our proposed design ( $p=1$, $q=1$ ) can reduce the blocking probability to $1.67 \times 10^{-4}$ [see Fig. 3(a)].

We also perform a simulation experiment on a single node under symmetric and asymmetric traffic, so that we can focus on the performance of the proposed node architecture. However, due to space limitation, we do not include the simulation results here. All these simulation results confirm that the proposed node architecture can achieve a very favorable tradeoff: its complexity can be significantly reduced while its performance only slightly degrades. For the special case when $p$ and $q$ equal $M$, our node architecture performs exactly the same as the SPN architecture.

\section{CONCLUSION}

This letter proposed a new node architecture called shared-by-wavelength-switches. This node architecture is more cost-effective than the existing node architectures because it requires much smaller and cheaper optical switches to achieve nearly the same blocking performance as the existing ones.

\section{REFERENCES}

[1] K. C. Lee and V. O. K. Li, "A wavelength convertible optical network," J. Lightw. Technol., vol. 11, no. 3, pp. 962-970, May/Jun. 1993.

[2] V. Eramo and M. Listanti, "Packet loss in a bufferless optical WDM switch employing shared tunable wavelength converters," J. Lightw. Technol., vol. 18, no. 12, pp. 1818-1833, Dec. 2000.

[3] — - "Input wavelength conversion in optical packet switches," IEEE Commun. Lett., vol. 7, no. 6, pp. 281-283, Jun. 2003.

[4] G. Xiao and Y. W. Leung, "Algorithms for allocating wavelength converters in all-optical networks," IEEE/ACM Trans. Netw., vol. 7, no. 4, pp. 545-557, Aug. 1999.

[5] V. Eramo, M. Listanti, and M. Spaziani, "Resources sharing in optical packet switches with limited-range wavelength converters," J. Lightw. Technol., vol. 23, no. 2, pp. 671-687, Feb. 2005.

[6] H. Li and I. L. J. Thng, "Performance analysis of a limited number of wavelength converters in an optical switching node," IEEE Photon. Technol. Lett., vol. 17, no. 5, pp. 1130-1132, May 2005.

[7] — "Cost-saving two-layer wavelength conversion in optical switching network," J. Lightw. Technol., vol. 24, no. 2, pp. 705-712, Feb. 2006.

[8] X. Chu, J. Liu, B. Li, and Z. Zhang, "Analytical model of sparse-partial wavelength conversion in wavelength-routed WDM networks," IEEE Commun. Lett., vol. 9, no. 1, pp. 69-71, Jan. 2005.

[9] J. Zheng and H. T. Mouftah, Optical WDM Networks Concepts and Design Principles. New York: Wiley, 2004.

[10] P. D. Dobbelaere, K. Falta, L. Fan, S. Gloeckner, and S. Patra, "Digital MEMS for optical switching," IEEE Commun. Mag., vol. 40, no. 3, pp. 88-95, Mar. 2002.

[11] D. J. Bishop, C. R. Giles, and G. P. Austin, "The Lucent LambdaRouter: MEMS technology of the future here today," IEEE Commun. Mag., vol. 40, no. 3, pp. 75-79, Mar. 2002.

[12] P. B. Chu, S. S. Lee, and S. Park, "MEMS: The path to large optical crossconnects," IEEE Commun. Mag., vol. 40, no. 3, pp. 80-87, Mar. 2002.

[13] R. Ramaswami and K. N. Sivarajan, Optical Networks A Practical Perspective, 2nd ed. San Mateo, CA: Morgan Kaufmann, 2002.

[14] P. H. Ho, H. T. Mouftah, and J. Wu, "A scalable design of multigranularity optical cross-connects for the next-generation optical internet," IEEE J. Sel. Areas Commun., vol. 21, no. 7, pp. 1133-1142, Sep. 2003. 